\title{
Hypercalcemia with progression to Acute Kidney Injury (AKI) after daily use of 120,000 UI of Vitamin D: a case report
}

\author{
Nayara Douat Hannegraf ${ }^{1}$, Isabelle Bolfe ${ }^{1}$, Renato Dalmagro ${ }^{1}$, Priscilla Dal Pra Campos ${ }^{2}$, Tatiana Rosa Ogata Nakagawa ${ }^{2}$ and Francelise \\ Bridi Cavassin ${ }^{3 *}$ \\ ${ }^{1}$ Medical Student; Medical School Undergraduate Program, Faculdades Pequeno Príncipe (FPP), Curitiba-PR, Brazil \\ ${ }^{2}$ Medical Doctor; Assistant Professor; Faculty of Medical Sciences, Faculdades Pequeno Príncipe (FPP), Curitiba-PR, Brazil \\ ${ }^{3}$ Pharmacist; Assistant Professor; Faculty of Medical Sciences, Faculdades Pequeno Príncipe (FPP), Curitiba-PR, Brazil
}

\begin{abstract}
Alternative therapies, such as the use of vitamin $\mathrm{D}$ in high daily doses, are increasingly common and available for clinical practice in the treatment of autoimmune diseases. Its use requires caution due to the active role of vitamin $\mathrm{D}$ in immunomodulation and calcium absorption by the body. Here we describe a case report about a condition of hypercalcemia with evolution to acute renal failure after using high daily doses of vitamin D as recommended by the alternative Coimbra Protocol. A twenty-one-years old, Caucasian female patient, presented with acute renal failure after continuous and daily use of 120,000 IU of cholecalciferol, according to medical recommendation. She has evolved with hypercalcemia and digestive symptoms due to intoxication by hypervitaminosis D. Due to refractory hypercalcemia despite the measures imposed, zoledronic acid was added to the therapy, which proved to be effective in resolving the case. The treatment of autoimmune diseases based on the Coimbra Protocol lacks studies that prove its effectiveness. Evidence is undoubtedly needed to prove the benefits and establish the risks to which patients may be exposed when using high daily doses of vitamin D.
\end{abstract}

\section{Introduction}

Rheumatoid arthritis (RA) is an autoimmune disease, of multifactorial etiology, characterized by chronic inflammation that involves not only the joints, but the synovial membrane and, eventually, extra-articular organs. [1,2] Therapy involves the association of nonsteroidal anti-inflammatory drugs (NSAIDs), corticosteroids and disease-modifying antirheumatic drugs (DMARDs). Such options may take time to exert their therapeutic effects and evolve with several side effects. For this reason, the search for alternative treatments has been intensified in recent decades.

In 2011, a new methodology called the Coimbra Protocol, which uses high daily doses of vitamin $\mathrm{D}$ to treat autoimmune diseases, began to be practiced [3]. Cholecalciferol plays an important role in immunomodulation, correlating inversely with disease activity [2]. Unlike what is found in healthy individuals, vitamin D receptors are present in the lymphocytes, macrophages, chondrocytes and synovial cells of RA patients [4]. This condition reflects the importance of vitamin $\mathrm{D}$ in the immune function and, as therefore, in the inflammatory response of the disease. The supplement promotes the absorption of calcium in the intestine and maintains adequate serum calcium and phosphate concentrations [1]. Its excess can lead to hypercalcemia, already observed in patients who use high doses of cholecalciferol $[5,6]$.

Considering practicing the Coimbra Protocol has gained significant space in the treatment of autoimmune diseases and that there is little data in the literature on the subject, it is essential to evaluate the possible effects of hypervitaminosis D due to its potential renal risk.

\section{Case Report}

We present a female patient, 21 years old, student, hospitalized on an emergency basis through medical referral, in a large private hospital, due to complaints of nausea and abdominal / pelvic pain. Previous morbid history of rheumatoid arthritis, gluten allergy and lactose intolerance. Continued daily use of cholecalciferol 120,000 IU for at least six months, in the morning, according to the recommendations of the Coimbra Protocol prescribed by her personal physician. The patient reported similar symptoms months before, with a previous record of hypercalcemia, showing improvement after discontinuing the supplement. Other symptoms reported were dry mouth, burning eyes, hair loss, fatigue, very concentrated and low volume urine.

Upon physical examination, the patient was in good general condition (BEG), lucid and oriented in time and space, eupneic, hydrated, pale, afebrile. On abdominal examination, hydro-air noises were reduced, with diffuse painful palpation, but without signs of peritoneal irritation. The lower limbs were free of edema and the calves

${ }^{*}$ Correspondence to: Cavassin FB, Pharmacist; Assistant Professor; Faculty of Medical Sciences, Faculdades Pequeno Príncipe (FPP), Curitiba-PR, Brazil; Post-Graduation Program in Internal Medicine and Health Sciences; Clinical Medicine Department, Complexo Hospital de Clínicas of Universidade Federal do Paraná (CHC/UFPR), Curitiba-PR, Brazil, Tel: +55 41 996961251; E-mail: fran_cavassin@yahoo.com.br

Key words: autoimmune diseases, coimbra protocol, hypervitaminosis D, acute renal failure

Received: March 25, 2021; Accepted: May 18, 2021; Published: May 21, 2021 
were free. Liquid diet, $0.9 \%$ saline, dimenhydrinate, ondansetron, dipyrone, pantoprazole were prescribed during this period. Laboratory analyzes revealed erythrocytes $\left(4.01 \times 106 / \mathrm{mm}^{3}\right)$ and globular volume (35.50\%) below the reference values. Erythrocyte sedimentation rateESR $(36 \mathrm{~mm})$, ferritin $(257 \mathrm{ng} / \mathrm{mL})$, uric acid $(8.0 \mathrm{mg} / \mathrm{dL})$, creatinineCr $(1.60 \mathrm{mg} / \mathrm{dL})$ and increased C-Reactive Protein-CRP $(10.3 \mathrm{mg} / \mathrm{L})$. Urinalysis showed cloudy urine, proteinuria, hematuria, leukocyturia and bacteriuria.

Also reported weight loss in the last month, with body mass indexBMI $\left(18 \mathrm{~kg} / \mathrm{m}^{2}\right)$ below ideal. Seriography revealed to be the patient with the Nutcracker Syndrome.

Subsequently, the patient evolved with mild abdominal discomfort, concentrated urine and low diuresis. New tests showed worsening of renal function, with $\mathrm{Cr}(1.7 \mathrm{mg} / \mathrm{dL})$ and glomerular filtration rate-GFR $(42 \mathrm{ml} / \mathrm{min})$. In addition, there was an increase in the values of ESR, CRP and positive rheumatoid factor-RF $(147.7 \mathrm{UI} / \mathrm{ml})$. The rheumatologist requested further tests to rule out autoimmune disease, which returned within normal standards. Only then was the dosage of serum calcium$\mathrm{Ca}^{++}$requested. On the fourth day (D4), hypercalcemia $\mathrm{Ca}^{++}(14.2 \mathrm{mg} /$ $\mathrm{dL}$ ) was confirmed secondary to intoxication due to the use of Vitamin $\mathrm{D}$, which evolved to acute renal failure (ARI) with digestive symptoms.

The treatment was carried out with intense intravenous hydration, furosemide and hydrocortisone, associated with fluid intake, with control and measurement of diuresis. Despite the conduct, the patient developed nausea and refractory hypercalcemia. It was then decided to administer zoledronic acid (4mg IV/15min), showing an adequate therapeutic response despite the appearance of some unwanted effects (fever, headache, myalgia, inappetence, fainting and chills).

Discharge was prescribed after clinical improvement, stable vital signs and adequate levels of calcemia $\mathrm{Ca}^{++}(5.08 \mathrm{mg} / \mathrm{dL})$ and $\mathrm{Cr}(0.9 \mathrm{mg} /$ $\mathrm{dL}$ ). The therapeutic plan at home was to maintain fluid intake (1.5 to $2.0 \mathrm{~L} /$ day), use of furosemide for a short period, weight control and diuresis.

\section{Discussion}

In this reported case, the patient had a confirmed diagnosis of RA and was using an alternative methodology for treatment, under continuous and daily use of cholecalciferol 120,000 IU, for at least six months. Vitamin D is a fundamental hormone for the control of calcium metabolism. If there is an imbalance in the regulation of this ion caused by its excessive renal retention, intestinal absorption and/ or bone resorption, hypercalcemia may occur with possible evolution to AKI.

As described, hypercalcemia was identified with worsening renal function due to vitamin $\mathrm{D}$ intoxication. The therapeutic approach was based on fluid replacement and the use of medications. Without resolution of the condition, zoledronic acid was added to the therapeutic regimen. This is a drug that belongs to the bisphosphonate class and acts by suppressing the activity of osteoclasts, inhibiting bone resorption.
That is, it causes less calcium to be transferred from the bones to the blood, decreasing the serum amount of ion and, consequently, reducing renal overload. As a result, calcium levels returned to normal standards allowing hospital discharge.

The use of high daily doses of vitamin $\mathrm{D}$, as recommended by the Coimbra Protocol, requires clinical studies that demonstrate its risks and benefits. In a try to better support the discussion, we found only two cases report both of a 70-year-old hypertensive and diabetic patient who developed similar signs and symptoms after using 6,00,000 IU every two weeks for the last two years for arthralgias and generalized body aches [7] and the other in use of 2000UI vitamin D3 / day associated with $0.25 \mathrm{mcg}$ of calcitriol [8]. The dose used by this patient was 60 times lower than that recommended by the protocol and even so, it was shown to cause kidney damage. The daily recommendation to supplement vitamin D is about 400-800 extendable to 2000 IU depends on age, body weight, disease-status, and ethnicity [9].

We believe this to be the first case report in the literature, as extensive research in national and international databases has not shown similar results using vitamin $\mathrm{D}$ supplementation with such a high daily dosage.

\section{Conclusion}

Alternative treatment of an autoimmune condition demonstrates the risks to which patients may be exposed even if the therapy line is a simple vitamin supplementation. Due to the important role of vitamin $\mathrm{D}$ in calcium absorption, its indication for use in high dosages should be performed with caution. Evidence that shows its effectiveness is necessary in order to avoid exposing individuals to high risky situations.

\section{References}

1. Meena N, Chawla SPS, Garg R, Batta A, Kaur S (2018) Assesment of Vitamin D in Rheumatoid Arthritis and Its Correlation with Disease Activity. J Nat Sci Biol Med 9: 54-58. [Crossref]

2. Bragazzi NL, Watad A, Neumann SG, Simon M, Brown SB, et al. (2017) Vitamin D and rheumatoid arthritis: an ongoing mystery. Curr Opin Rheumatol 29: 378-388. [Crossref]

3. https://vitaminadporumaoutraterapia.wordpress.com/about/.

4. Hansen KE, Bartels CM, Gangnon RE, Jones AN, Gogineni J (2014) An evaluation of high-dose vitamin D for rheumatoid arthritis. J Clin Rheumatol 20: 112-114. [Crossref]

5. Lowe H, Cusano NE, Binkley N, Blaner WS, Bilezikian JP (2011) Vitamin D Toxicity due to a Commonly Available "Over the Counter" Remedy from the Domincan Republic. J Clin Endocrinol Metab 96: 291-295. [Crossref]

6. Aihara S, Yamada S, Oka H, Kamimura T, Nakano T, et al. (2019) Hypercalcemia and acute kidney injury induced by eldecalcitol in patients with osteoporosis: a case series of 32 patients at a single facility. Renal Falilure 41: 88-97. [Crossref]

7. Naik MA, Banday KA, Najar MS, Reshi AR, Bhat MA (2008) Vitamin D intoxication presenting as acute renal failure. Indian J Nephrol 18: 125-126. [Crossref]

8. Guerra V, Neto OMV, Laurindo AF, De Paula FJA, Neto MM (2016) Hipercalcemia e prejuízo de função renal associados à intoxicação por vitamina D: relato de caso. $J$ Bras Nefrol 38: 466-469.

9. https://doi.org/10.1016/j.jsbmb.2017.01.021.

Copyright: (C2021 Hannegraf ND. This is an open-access article distributed under the terms of the Creative Commons Attribution License, which permits unrestricted use, distribution, and reproduction in any medium, provided the original author and source are credited. 